\title{
From strangelets to strange stars: A unified description
}

\author{
Cheng-Jun Xia · Guang-Xiong Peng · En-Guang Zhao · Shan-Gui Zhou
}

the date of receipt and acceptance should be inserted later

\begin{abstract}
The conventionally separated treatments for strangelets and strange stars are now unified with a more comprehensive theoretical description for objects ranging from strangelets to strange stars. After constraining the model parameter according to the Witten-Bodmer hypothesis and observational massradius probability distribution of pulsars, we investigate the properties of this kind of objects. It is found that the energy per baryon decreases monotonically with increasing baryon number and reaches its minimum at the maximum baryon number, corresponding to the most massive strange star. Due to the quark depletion, an electric potential well is formed on the surface of the quark part. For a rotational bare strange star, a magnetic field with the typical strength in pulsars is generated.
\end{abstract}

Keywords strangelets · strange stars · strange quark matter · unified description · Witten-Bodmer hypothesis

C.-J. Xia ${ }^{1}$, E-mail: cjxia@itp.ac.cn · G.-X. Peng ${ }^{2,3,4}$. E.-G. Zhao $^{1,5}$. S.-G. Zhou ${ }^{1,4,5}$

${ }^{1}$ State Key Laboratory of Theoretical Physics, Institute of Theoretical Physics, Chinese Academy of Sciences, Beijing 100190, China

${ }^{2}$ School of Physics, University of Chinese Academy of Sciences, Beijing 100049, China

${ }^{3}$ Theoretical Physics Center for Science Facilities, Institute of High Energy Physics, Beijing 100049, China

${ }^{4}$ Synergetic Innovation Center for Quantum Effects and Application, Hunan Normal University, Changsha 410081, China ${ }^{5}$ Center of Theoretical Nuclear Physics, National Laboratory of Heavy Ion Accelerator, Lanzhou 730000, China

\section{Introduction}

It was pointed out long ago that strange quark matter (SQM) might be the ground state of strongly interacting matter, which is nowadays called the WittenBodmer hypothesis 1.2. If true, there should exist stable objects of SQM with the baryon number $A$ ranging from a few to $\sim 10^{57}$. Customarily, small SQM nuggets with $A \lesssim 10^{7}$ are often referred to as strangelets 3 - 10 , or slets [11, while stars consisting of SQM are called strange (quark) stars 12 21, being possible candidates for pulsars.

Lumps of SQM are expected to be produced in the collision of binary compact stars containing SQM 22 23. Further collisions among those lumps may create slets, nuclearites 24 25, meteorlike compact ultradense objects [26] etc., and some of them may eventually make their way to our Earth 27]. Due to the special characteristics of these objects such as the lower chargeto-mass ratio 28, 29, the larger mass [30, the highly ionizing tracks in the interstellar hydrogen cloud (e.g., pulsar scintillations) [31, and the characteristic gamma rays through heavy ion activation 32 , there are possibilities to observe them. However, despite decades of efforts, no compelling evidence for the existence of stable SQM is found (for reviews, see, e.g., Refs. [33, 34]).

This case is due to the extreme complexity of an SQM system which involves all the fundamental interactions, i.e., the strong, weak, electromagnetic, and gravitational interactions. In the conventional theoretical treatments, significantly different simplifications were adopted for slets and strange stars. For a slet, electrons were ignored since the Compton wavelength is much larger than the size of the quark part 35, and quarks were assumed to be uniformly distributed. For strange stars, gravity has to be considered. The nor- 
mal way is to first get an equation of state of SQM by assuming the local charge neutrality, and then obtain the mass-radius $(M-R)$ relation by solving the TolmanOppenheimer-Volkov equations.

However, according to recent studies, effects such as the charge screening, electron-positron pair creation, and nonzero charge densities in strange stars have important implications on the properties of SQM. For example, taking into account the electrostatic effects, Alford et al. 36 found that, for a small enough surface tension, large slets are unstable to fragmentation and strange star surfaces fragment into a crystalline crust made of slets and electrons. For quark-hadron phase transition, the finite-size effect turns out to be very important 3740 . It was shown that the geometrical structures may be destabilized by the charge screening effect 41]. Due to the electron-positron pair creation, an upper bound on the net charge of slets or strange stars was found 42]. The local charge neutrality in compact stars is also in question [43. In the case of a neutron star, an overcritical electric field was found in the transitional region from the core to the crust [44]. For a bare strange star, an electric dipole layer may be formed on the surface and results in an electric field of $\sim 10^{17-19} \mathrm{~V} / \mathrm{cm}$ 12. Due to the presence of a critical electric field, the electron-positron production may be induced and results in some astrophysical observables 45 . The mass and radius of a strange star are increased by $\sim 15 \%$ and $\sim 5 \%$, respectively, if the star possesses a net charge on the surface 46 .

Meanwhile, the possibility of pulsars being strange stars may give us an insight into the properties of SQM. Up till now, around 2,500 pulsars have been observed and among them about 70 pulsars' masses were measured 47,48. At the same time, more than 10 pulsars provide us the $M-R$ probability distributions with photospheric radius expansion bursts as well as quiescent low-mass X-ray binaries 49 53. If SQM is absolutely stable, those pulsars may be strange stars [54, then the properties of SQM can be constrained with the $M-R$ relations.

In the present paper, we study the SQM system ranging from slets to strange stars in a unified description. After constraining the only model parameter, the bag constant $B$, according to the Witten-Bodmer hypothesis and the observational $M-R$ probability distribution of pulsars, it is found that the ratio of charge to baryon number of a slet is different from previous findings, while the size is significantly smaller than that of a nucleus with the same mass number. In addition, rotation of a bare strange star generates a strong magnetic field with the typical strength in pulsars. This paper is organized as follows. In Section 2, the uni- fied description for SQM objects is presented, where the effects of gravity and electrostatic interactions are treated on the macroscopic scale while the strong and weak interactions are considered locally. Based on this description, the properties of SQM objects ranging from slets to strange stars are investigated in Section 3 A summary is presented in Section 4

\section{Theoretical framework}

The internal structure of a spherically symmetric, charged, and static object should fulfill the thermodynamic equilibrium condition, which can be obtained by minimizing the energy of the system for given total particle number and entropy. We consider the gravity and electrostatic interactions on the macroscopic scale. The metric for the SQM sphere reads

$\mathrm{d} s^{2}=\mathrm{e}^{\nu} \mathrm{d} t^{2}-\mathrm{e}^{\lambda} \mathrm{d} r^{2}-r^{2}\left(\mathrm{~d} \theta^{2}+\sin ^{2} \theta \mathrm{d} \phi^{2}\right)$,

where $r, \theta$, and $\phi$ are the standard spherical coordinates with the metric elements satisfying

$$
\begin{aligned}
\mathrm{e}^{-\lambda} & =1-\frac{2 G}{r} M_{\mathrm{t}}, \\
\frac{\mathrm{d} \nu}{\mathrm{d} r} & =\frac{2 G \mathrm{e}^{\lambda}}{r^{2}}\left[4 \pi r^{3}\left(P-\frac{\alpha Q^{2}}{8 \pi r^{4}}\right)+M_{\mathrm{t}}\right] .
\end{aligned}
$$

Here we use the natural system of units, with $G$ and $\alpha$ being the gravitational and fine-structure constants. The total mass, particle number, and entropy are obtained with

$$
\begin{aligned}
M_{\mathrm{t}}(r) & =\int_{0}^{r} 4 \pi r^{\prime 2}\left(E+\alpha Q^{2} / 8 \pi r^{4}\right) \mathrm{d} r^{\prime}, \\
N_{i}(r) & =\int_{0}^{r} 4 \pi n_{i}\left(r^{\prime}\right) \mathrm{e}^{\lambda / 2} r^{\prime 2} \mathrm{~d} r^{\prime}, \\
\bar{S}(r) & =\int_{0}^{r} 4 \pi S\left(r^{\prime}\right) \mathrm{e}^{\lambda / 2} r^{\prime 2} \mathrm{~d} r^{\prime} .
\end{aligned}
$$

Then the total charge is given by $Q(r)=\sum_{i} q_{i} N_{i}(r)$ with $q_{u}=2 / 3, q_{d}=q_{s}=-1 / 3$, and $q_{e}=-1$. Based on the Thomas-Fermi approximation, the pressure $P(r)$, energy density $E(r)$, particle number density $n_{i}(r)$, and entropy density $S(r)$ are given locally by incorporating both the strong and weak interactions.

By minimizing the mass $M=M_{\mathrm{t}}(\infty)$ with respect to the particle distribution $N_{i}(r)$ and entropy distribution $\bar{S}(r)$ at the fixed total particle number $N_{i}(\infty)$ and entropy $\bar{S}(\infty)$, we immediately have

$$
\begin{aligned}
\frac{\mathrm{d} \mu_{i}}{\mathrm{~d} r} & =\frac{Q}{r^{2}} q_{i} \alpha \mathrm{e}^{\lambda / 2}-\frac{\mu_{i}}{2} \frac{\mathrm{d} \nu}{\mathrm{d} r}, \\
\frac{\mathrm{d} T}{\mathrm{~d} r} & =-\frac{T}{2} \frac{\mathrm{d} \nu}{\mathrm{d} r},
\end{aligned}
$$

with $\mu_{i}(r)$ and $T(r)$ being the chemical potential and temperature. 
For the local properties of SQM, we adopt the bag model and consider only zero temperature, where the thermodynamic potential density is given by

$\Omega(r)=\Omega_{0}\left(\left\{\mu_{i}(r)\right\},\left\{m_{i}\right\}\right)+B$

in the ideal Fermi-gas approximation. To reach the lowest energy, SQM undergoes weak reactions and reach the chemical equilibrium

$\mu_{u}+\mu_{e}=\mu_{d}=\mu_{s}$.

Then the internal structure of an SQM sphere can be determined by solving Eq. (7). Since electrons are not confined by the vacuum pressure, an atom-like structure of the SQM system is formed, i.e., a positively charged SQM core with a cloud of electrons surrounding it.

The quark-vacuum interface on the surface of the SQM core needs to be treated with special care. We consider the number of depleted quarks on the interface by adopting the multiple reflection expansion (MRE) method [3, 4

$\frac{\mathrm{d} N_{i}^{\text {surf }}}{\mathrm{d} p_{i}}=\frac{2 g_{i} R}{3 \pi}-\frac{g_{i} p_{i} R}{m_{i} \pi}\left(m_{i} R+1\right) \arctan \left(\frac{m_{i}}{p_{i}}\right)$.

Here $p_{i}$ is the momentum of quark flavor $i(i=u, d, s)$. Its upper bound corresponds to the Fermi momentum on the surface $\nu_{i}(R)$ with $R$ being the radius of the SQM core. Note that Eq. 11 only gives the average number of depleted quarks, while for smaller systems shell corrections may be important [55]. Then the energy contribution and pressure are given by

$\bar{E}_{i}^{\text {surf }}=\int_{0}^{\nu_{i}(R)} \sqrt{p_{i}^{2}+m_{i}^{2}} \frac{\mathrm{d} N_{i}^{\text {surf }}}{\mathrm{d} p_{i}} d p_{i}$

and

$P^{\text {surf }}=-\left.\sum_{i} \frac{\mathrm{d} \bar{E}_{i}^{\text {surf }}}{\mathrm{d} V}\right|_{N_{i}^{\text {surf }}}$.

Under the influence of gravity, the energy contribution to the mass is $M^{\text {surf }}=\sum_{i} \bar{E}_{i}^{\text {surf }} \mathrm{e}^{\nu(R) / 2}$.

The quark-vacuum interface is obtained when the pressure of quarks is in balance with the vacuum pressure, i.e.,

$P(R)-P_{e}(R)+P^{\text {surf }}=0$.

Then we have the total quark number $N_{q}=N_{q}(R)+$ $N_{q}^{\text {surf }}$, mass $M=M_{\mathrm{t}}(\infty)+M^{\text {surf }}$, and charge of the core $Q(R)=\sum_{i} q_{i}\left[N_{i}(R)+N_{i}^{\text {surf }}\right]$.

\section{Results and discussions}

For a given core radius $R$, the structure of an SQM sphere is determined by solving the differential equation (7) under the boundary conditions, i.e., $M_{\mathrm{t}}(0)=0$,

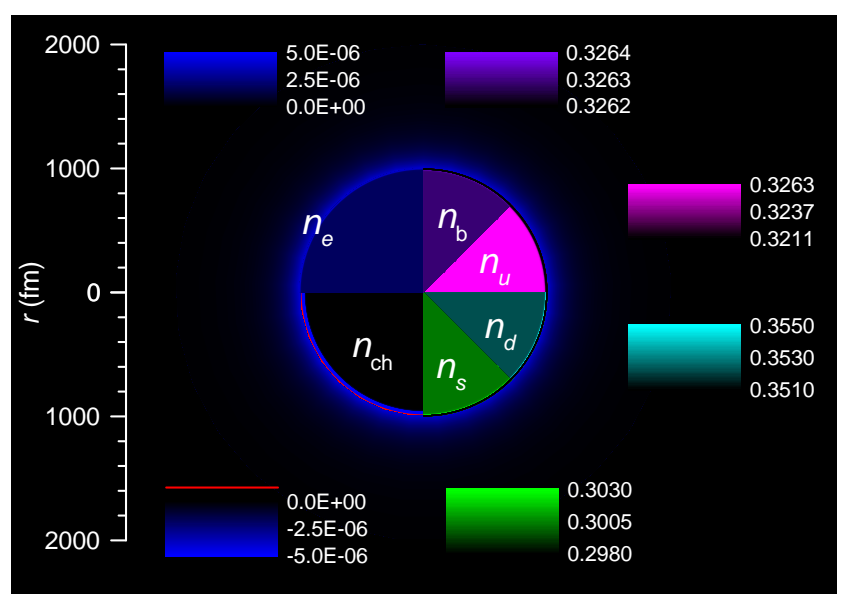

Fig. 1 (Color online) The density profiles (in $\mathrm{fm}^{-3}$ ) of an SQM sphere with the core radius $R=1000 \mathrm{fm}$, where the bag constant is taken as $B^{1 / 4}=152 \mathrm{MeV}$. Here $n_{\mathrm{ch}}=$ $\sum_{i} q_{i} n_{i}$ corresponds to the local charge density, while $n_{\mathrm{b}}=$ $\sum_{q=u, d, s} n_{q} / 3$ is the local baryon number density.

$Q(0)=Q(\infty)=0$, and Eq. (14). To illustrate our results, we present a colored contour plot in Fig. 1 for an SQM sphere with the core radius $R=1000 \mathrm{fm}$. A rich charge profile on the surface is found. At the $r>R$ region, there exists an electron cloud which neutralizes the positively charged core and expands by $\sim 1 \AA$. Respectively, the SQM sphere contains $1.37 \times 10^{9}$ $u$-quarks, $1.48 \times 10^{9} \mathrm{~d}$-quarks, $1.26 \times 10^{9} s$-quarks, and $2.74 \times 10^{5}$ electrons, which gives the total core charge $Q(R)=2.66 \times 10^{5}$ and mass $M=1.22 \times 10^{12}$ $\mathrm{MeV}$. Due to the quark depletion on the core surface $\left(N_{u}^{\text {surf }}=-3.34 \times 10^{4}, N_{d}^{\text {surf }}=-7.06 \times 10^{4}\right.$, and $\left.N_{s}^{\text {surf }}=-1.03 \times 10^{6}\right)$, there exists a surface charge $Q^{\text {surf }}=\sum_{i} q_{i} N_{i}^{\text {surf }}=3.43 \times 10^{5}$, while the corresponding mass modification is $M^{\text {surf }}=-2.15 \times 10^{8} \mathrm{MeV}$.

Note that the current masses of quarks and the electron mass are taken as $m_{u}=2.3 \mathrm{MeV}, m_{d}=4.8 \mathrm{MeV}$, $m_{s}=95 \mathrm{MeV}$, and $m_{e}=0.511 \mathrm{MeV}$ [56], leaving only the bag constant $B$ undetermined. For SQM to stably exist at zero external pressure, the bag constant should meet the requirement of the Witten-Bodmer hypothesis, which gives $144.37<B^{1 / 4}<159.26 \mathrm{MeV}$. We take the three typical values $B^{1 / 4}=145,152$, and 159 $\mathrm{MeV}$. It is worth mentioning that if $B$ exceeds the upper bound, SQM is unstable and may only exist at the core of a compact star, i.e., hybrid star 13 .57.

A full calculation from slets to strange stars is carried out. In Fig. 2, the energy per baryon is given, which decreases monotonously with increasing radius or baryon number. When the energy per baryon reaches $930 \mathrm{MeV}$, the minimum baryon numbers for absolutely stable slets are determined, i.e., $A_{\min }=24,80$, and 394849 , which increases dramatically as $B$ approaches 


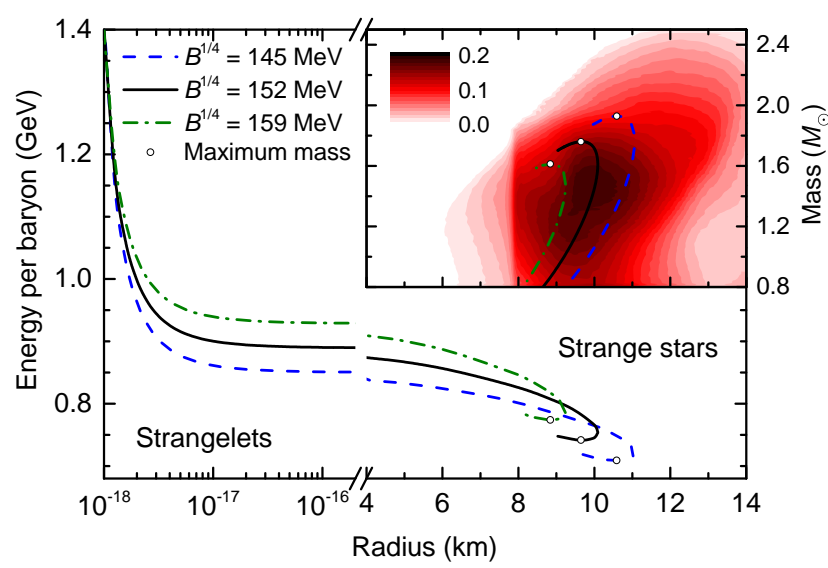

Fig. 2 (Color online) The energy per baryon from slets to strange stars. In the inset, the obtained $M-R$ relations are compared with the normalized $M-R$ probability distribution compiled from the observed values of eight pulsars 49 . Fig. 10].

to its upper limit. In the region with $200 \mathrm{fm} \lesssim R \lesssim$ $1 \mathrm{~km}$, the variation of the energy per baryon is infinitesimal, and the radius is related to the baryon number by $R=r_{0} A^{1 / 3}$ with $r_{0}=0.944,0.901$, and $0.862 \mathrm{fm}$. When $R \gtrsim 1 \mathrm{~km}$, gravity starts to reduce the energy per baryon and a minimum value is obtained, corresponding to the maximum mass and baryon number of strange stars. The obtained $M-R$ relations, as shown in the inset of Fig. 2, are in good agreement with the $M-R$ probability distribution obtained by averaging the observed values of eight pulsars [4, Fig. 10].

The charge-to-mass ratio in Fig. 3(a) is defined as $f_{Z} \equiv Q(R) / A$ ignoring the electrons surrounding the SQM core. The obtained values are larger than previous results 3,58. For smaller $B$, the SQM core carries slightly more charge. Note that when $R \lesssim 13 \mathrm{fm}$, SQM within slets is positively charged. However, for $R \gtrsim 17 \mathrm{fm}$, as shown in Fig. 1, the SQM carries negative charge to compensate the positive surface charge. Then an electric potential well for negatively charged particles is formed due to the quark depletion on the quarkvacuum interface. These particles may be trapped in the potential well and give a distinct photon spectrum when excited, which have significant implications for the experimental searches of SQM. As indicated in Fig. 3(b), when $R \gtrsim 10^{5} \mathrm{fm}$, charges are mostly located on the core surface and can be described by a constant surface charge density $\sigma$ with $Q(R)=\sigma R^{2}$. It is found that $\sigma=0.0144,0.0135$, and $0.0128 \mathrm{fm}^{-2}$ for $B^{1 / 4}=145$, 152 , and $159 \mathrm{MeV}$, which are much larger than the upper bound $7 \times 10^{-5} \mathrm{fm}^{-2}$ considering the electronpositron pair creation 42].

When the SQM core rotates, a magnetic field may be generated. For strong enough field strength, the elec-

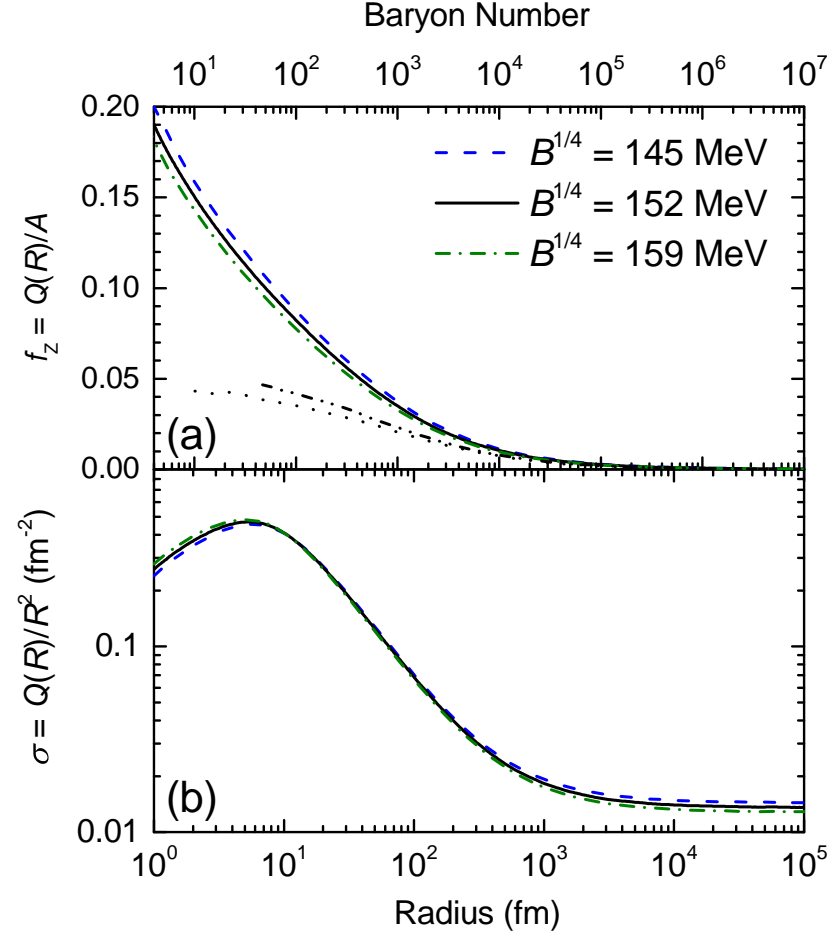

Fig. 3 (Color online) (a): The charge-to-mass ratio of the SQM core of slets, which is compared with previous findings indicated by the dotted line [3, Fig. 4] and dash-dot-dotted line 58. Fig. 2]; (b): The surface charge density of the SQM core as functions of radius.

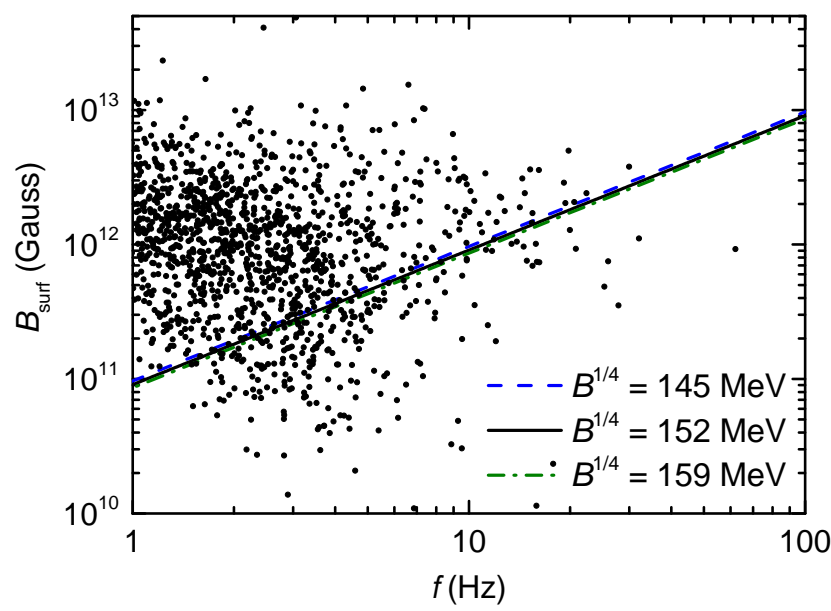

Fig. 4 (Color online) The magnetic field of rotating strange stars estimated with Eq. 15. The dots depict the inferred magnetic fields of pulsars obtained from ATNF Pulsar Catalogue 47, 48, where the binary pulsars are excluded since their magnetic fields may be dampened by mass accretion 59]. 
trons are expected to be trapped along the magnetic field lines. Then we simply assume the electron cloud stays still while the core rotates. It is straightforward to write out the magnetic field at the pole area on the surface 60]:

$B_{\text {surf }}=\frac{1}{3} u_{0} \sigma R f$.

Here $u_{0}$ represents the vacuum permeability while $f$ is the rotational frequency. Then in Fig. 4 the magnetic field of a rotating strange star is obtained, with the field strength approaching to the typical value of pulsars, e.g., $\sim 10^{12}$ Gauss at $f=10 \mathrm{~Hz}$. It is found that the variation of the bag constant $B$ barely affects the field strength.

\section{Summary}

In conclusion, we propose a unified description from strangelets to strange stars considering the gravity and electrostatic interactions on the macroscopic scale while incorporating the strong and weak interactions locally. The quark-vacuum interface is treated with the multiple reflection expansion method. The model parameter follows the Witten-Bodmer hypothesis and is confronted with the observational $M-R$ probability distributions of pulsars. Then the properties of SQM systems with all possible baryon numbers are investigated. It is found that the energy per baryon decreases monotonously for increasing baryon number, while the obtained charge-to-mass ratio of the SQM core is larger than previous predictions. On the core surface, due to the quark depletion, an electric potential well is formed for negatively charged particles and may give some unique observables for SQM detection. When $R \gtrsim 10^{5}$ $\mathrm{fm}$, charges are mostly located on the core surface and a constant surface charge density is obtained. Then for a rotational bare strange star, a magnetic field comparable to the typical strength of pulsars is generated.

Acknowledgements We are grateful to Professors LieWen Chen, Thomas Papenbrock, Michael Smith, and SheSheng Xue for fruitful discussions. This work was supported by National Natural Science Foundation of China (Grant Nos. 11135011, 11120101005, 11275248, 11475110, 11475115, 11575190 and 11525524), National Key Basic Research Program of China (Grant No. 2013CB834400), and the Knowledge Innovation Project of the Chinese Academy of Sciences (Grant No. KJCX2-EW-N01). The computation of this work was supported by the HPC Cluster of SKLTP/ITP-CAS and the Supercomputing Center, CNIC of CAS.

\section{References}

1. Bodmer A R. Collapsed Nuclei. Phys Rev D, 1971, 4: 1601-1606
2. Witten E. Cosmic separation of phases. Phys Rev D, 1984, 30: 272-285

3. Berger M S, Jaffe R L. Radioactivity in strange quark matter. Phys Rev C, 1987, 35: 213-225

4. Madsen J. Curvature contribution to the mass of strangelets. Phys Rev Lett, 1993, 70: 391-393

5. Farhi E, Jaffe R L. Strange matter. Phys Rev D, 1984, 30: 2379-2390

6. Greiner C, Koch P, Stöcker H. Separation of strangeness from antistrangeness in the phase transition from quark to hadron matter: Possible formation of strange quark matter in heavy-ion collisions. Phys Rev Lett, 1987, 58: $1825-1828$

7. Gilson E P, Jaffe R L. Stability of very small strangelets. Phys Rev Lett, 1993, 71: 332-335

8. He Y B, Gao C S, Li X Q, et al. Properties of strangelets at finite temperature in the liquid drop model. Phys Rev C, 1996, 53: 1903-1910

9. Wen X J, Peng G X, Chen Y D. Charge, strangeness and radius of strangelets. J Phys G: Nucl Part Phys, 2007, 34: 1697

10. Xia C J, Chen S W, Peng G X. Properties of strangelets in a new quark mass confinement model with one-gluonexchange interaction. Sci Chin Phys Mech Astron, 2014, 57: 1304

11. Peng G X, Wen X J, Chen Y D. New solutions for the color-flavor locked strangelets. Phys Lett B, 2006, 633: 314-318

12. Alcock C, Farhi E, Olinto A. Strange stars. Astrophys J, 1986, 310: 261-272

13. Weber F. Strange quark matter and compact stars. Prog Part Nucl Phys, 2005, 54: 193-288

14. Itoh N. Hydrostatic Equilibrium of Hypothetical Quark Stars. Prog Theor Phys, 1970, 44: 291-292

15. Haensel P, Zdunik J L, Schaeffer R. Strange quark stars. Astron Astrophys, 1986, 160: 121-128

16. Perez-Garcia M A, Silk J, Stone J R. Dark Matter, Neutron Stars, and Strange Quark Matter. Phys Rev Lett, 2010, 105: 141101

17. Herzog M, Röpke F K. Three-dimensional hydrodynamic simulations of the combustion of a neutron star into a quark star. Phys Rev D, 2011, 84: 083002

18. Dexheimer V, Torres J, Menezes D. Stability windows for proto-quark stars. Eur Phys J C, 2013, 73: 2569

19. Chang Q, Chen S W, Peng G X, et al. Properties of colorflavor locked strange quark matter and strange stars in a new quark mass scaling. Sci Chin Phys Mech Astron, 2013, 56: 1730

20. Xia C J, Peng G X, Chen S W, et al. Thermodynamic consistency, quark mass scaling, and properties of strange matter. Phys Rev D, 2014, 89: 105027

21. Xu J F, Peng G X, Liu F, et al. Strange matter and strange stars in a thermodynamically self-consistent perturbation model with running coupling and running strange quark mass. Phys Rev D, 2015, 92: 025025

22. Madsen J. Strangelet propagation and cosmic ray flux. Phys Rev D, 2005, 71: 014026

23. Bauswein A, Janka H T, Oechslin R, et al. Mass Ejection by Strange Star Mergers and Observational Implications. Phys Rev Lett, 2009, 103: 011101

24. Rújula A D, Glashow S L. Nuclearites-a novel form of cosmic radiation. Nature, 1984, 312: 734

25. Lowder D M. Experimental searches for strange quark matter in cosmic rays. Nucl Phys B (Proc Suppl), 1991, 24: $177-183$

26. Rafelski J, Labun L, Birrell J. Compact Ultradense Matter Impactors. Phys Rev Lett, 2013, 110: 111102 
27. Monreal B. Cosmic-ray strangelets in the Earth's atmosphere. J High Energy Phys, 2007, 02: 077

28. Sandweiss J. Overview of strangelet searches and Alpha Magnetic Spectrometer: when will we stop searching? J Phys G: Nucl Part Phys, 2004, 30: S51

29. Han K, Ashenfelter J, Chikanian A, et al. Search for Stable Strange Quark Matter in Lunar Soil. Phys Rev Lett, 2009, 103: 092302

30. Herrin E T, Rosenbaum D C, Teplitz V L. Seismic search for strange quark nuggets. Phys Rev D, 2006, 73: 043511

31. Pérez-García M Á, Silk J, Pen U L. Pulsar scintillation patterns and strangelets. Phys Lett B, 2013, 727: 357

32. Isaac M C P, Chan Y D, Clark R, et al. Search for Strange Matter by Heavy Ion Activation. Phys Rev Lett, 1998, 81: $2416-2419$

33. Klingenberg R. Strange quark matter searches. J Phys G: Nucl Part Phys, 1999, 25: R273

34. Finch E. Strangelets: who is looking and how? J Phys G: Nucl Part Phys, 2006, 32: S251

35. Madsen J. Physics and astrophysics of strange quark matter. In Lecture Notes in Physics, volume 516, pages 162-203, edited by Cleymans J, Geyer H, Scholtz F. Springer Berlin Heidelberg, 1999

36. Alford M G, Rajagopal K, Reddy S, et al. Stability of strange star crusts and strangelets. Phys Rev D, 2006, 73: 114016

37. Voskresensky D, Yasuhira M, Tatsumi T. Charge screening in hadron-quark mixed phase. Phys Lett B, 2002, 541: 93-100

38. Tatsumi T, Yasuhira M, Voskresensky D. Hadron-quark mixed phase in neutron stars. Nucl Phys A, 2003, 718: 359-362

39. Voskresensky D, Yasuhira M, Tatsumi T. Charge screening at first order phase transitions and hadron-quark mixed phase. Nucl Phys A, 2003, 723: 291-339

40. Endo T, Maruyama T, Chiba S, et al. Numerical study of the hadron-quark mixed phase. Nucl Phys A, 2005, 749: 333-336

41. Maruyama T, Chiba S, Schulze H J, et al. Hadron-quark mixed phase in hyperon stars. Phys Rev D, 2007, 76: 123015

42. Madsen J. Universal Charge-Radius Relation for Subatomic and Astrophysical Compact Objects. Phys Rev Lett, 2008, 100: 151102

43. Rotondo M, Rueda J A, Ruffini R, et al. The selfconsistent general relativistic solution for a system of degenerate neutrons, protons and electrons in $\beta$ equilibrium. Phys Lett B, 2011, 701: 667-671

44. Belvedere R, Pugliese D, Rueda J A, et al. Neutron star equilibrium configurations within a fully relativistic theory with strong, weak, electromagnetic, and gravitational interactions. Nucl Phys A, 2012, 883: 1-24

45. Ruffini R, Vereshchagin G, Xue S S. Electron-positron pairs in physics and astrophysics: From heavy nuclei to black holes. Phys Rep, 2010, 487: 1-140

46. Negreiros R P, Weber F, Malheiro M, et al. Electrically charged strange quark stars. Phys Rev D, 2009, 80: 083006

47. Manchester R N, Hobbs G B, Teoh A, et al. The Australia Telescope National Facility Pulsar Catalogue. Astron J, 2005, 129: 1993

48. http://www.atnf.csiro.au/research/pulsar/psrcat/ ATNF Pulsar Catalogue

49. Lattimer J M. The Nuclear Equation of State and Neutron Star Masses. Annu Rev Nucl Part Sci, 2012, 62: $485-515$
50. Steiner A W, Lattimer J M, Brown E F. The Equation of State from Observed Masses and Radii of Neutron Stars. Astrophys J, 2010, 722: 33

51. Guillot S, Servillat M, Webb N A, et al. Measurement of the Radius of Neutron Stars with High Signal-to-noise Quiescent Low-mass X-Ray Binaries in Globular Clusters. Astrophys J, 2013, 772: 7

52. Lattimer J, Steiner A. Constraints on the symmetry energy using the mass-radius relation of neutron stars. Eur Phys J A, 2014, 50: 40

53. Li Z S, Qu Z J, Chen L, et al. An Ultra-low-mass and Small-radius Compact Object in 4U 1746-37? Astrophys J, 2015, 798: 56

54. Page D, Reddy S. Dense Matter in Compact Stars: Theoretical Developments and Observational Constraints. Annu Rev Nucl Part Sci, 2006, 56: 327-374

55. Madsen J. Shell model versus liquid drop model for strangelets. Phys Rev D, 1994, 50: 3328-3331

56. Particle Data Group. Review of Particle Physics. Chin Phys C, 2014, 38: 090001

57. Wang H Y, Liu G Z, Wu Y R, et al. The third family of compact stars with the color-flavor locked quark core. Chinese Sci Bull, 2013, 58: 3731

58. Heiselberg H. Screening in quark droplets. Phys Rev D, 1993, 48: 1418-1423

59. Bhattacharya D, van den Heuvel E P J. Formation and evolution of binary and millisecond radio pulsars. Phys Rep, 1991, 203: 1-124

60. Negreiros R P, Mishustin I N, Schramm S, et al. Properties of bare strange stars associated with surface electric fields. Phys Rev D, 2010, 82: 103010 